\title{
Rifaximin for Treatment of Small Bowel Bacterial Overgrowth
}

\author{
Dabo Xu and Chung Owyang* \\ Division of Gastroenterology and Hepatology, Department of Internal Medicine, University of Michigan, Michigan, USA
}

Received: 此 September 03, 2018; Published: 制 September 06, 2018

*Corresponding author: Chung Owyang MD, Division of Gastroenterology, Department of Internal Medicine, University of Michigan, 3912 Taubman Center, Ann Arbor, MI 48109-5362, Michigan, USA

\begin{abstract}
Small intestinal bacterial overgrowth (SIBO) is often secondary to intestinal dysmotility and several other predisposing conditions. Treatment of SIBO typically includes antibiotics and treatment of underlying etiology. Identification and treatment of the underlying modifiable causes is critical for management of refractory and recurrent SIBO. We report here a case of scleroderma with small bowel dysmotility and bacterial overgrowth, on different occasions the patient failed to respond to Rifaximin. We showed that restoring small bowel motility is critical in maintaining the effectiveness of Rifaximin in the treatment of SIBO. Rifaximin resistance may also develop following prolonged use of antibiotics. Rotation of antibiotic regimen is important to prevent drug resistance.
\end{abstract}

Keywords: Breath Test; Case Report; Gut Microbiota; Irritable Bowel Syndrome; Nonabsorbable Antibiotics; Octreotide; Prokinetic Agents; Small Intestinal Bacterial Overgrowth

Abbreviations: CI: confidence Interval; FODMAPs: Fermentable Oligo, Di, And Monosaccharides and Polyols; HBT: Hydrogen Breath Test; HE: Hepatic Encephalopathy; IBS: Irritable Bowel Syndrome; SIBO: Small Intestinal Bacterial Overgrowth

\section{Introduction}

Small intestinal bacterial overgrowth (SIBO) is characterized by excessive bacteria in the small intestine. The upper small intestine is relatively sterile because of the combined effects of normal peristalsis and the antibacterial action of bile acids. Bacterial overgrowth may occur as a result of anatomic disturbances of the small intestine such as blind loops or motility disorders such as scleroderma [1]. However, the condition may also occur in the absence of any specific predisposing factor. Antibiotics are the mainstay of therapy for SIBO. Depending in underlying conditions, the choice, dosing and duration of antibiotic therapy may vary. Here we report a challenging case of recurrent and refractory SIBO associated with scleroderma where identification and treatment of the underlying small bowel motility disorder is critical. Furthermore because of the underlying dysmotility, this patient requires rotating antibiotic treatment whereby repeat glucose breath testing is needed to monitor recurrence and eradication of SIBO.

\section{Case Report}

An 18-year-old woman with a history of undifferentiated connective tissue disorder (scleroderma), Hashimoto's thyroiditis, celiac disease, and gastrointestinal dysmotility was admitted to the
UM Mott's Children's Hospital with worsening of abdominal pain, bloating, nausea and intolerance to enteral nutrition for 2 weeks. During the past year, patient was not able to tolerate oral intake and required enteral nutrition feeding via a gastrojejunostomy tube. Prior to the hospitalization, a glucose hydrogen breath test (HBT) was positive for hydrogen production with breath hydrogen increased form a basal of $5 \mathrm{ppm}$ to a peak of $231 \mathrm{ppm}$ following glucose challenge. Methane production was negative. She completed a 14 days' course of rifaximin (550 mg three times daily) without symptomatic improvement. Following admission, a repeat glucose HBT was again abnormal with a hydrogen rise of $218 \mathrm{ppm}$. Antroduodenal manometry showed intermittent bursts of uncoordinated contractile activities and infrequent occurrence of phase 3 migrating moro complexes (mmc) (1 mmc over $3 \mathrm{hr}$ ) during fasting, indicating a partial neurodegenerative process. Acute administration of octreotide (75 mcg, subcutaneously) evoked duodenal phase III complexes similar to those observed in normal subjects. Subsequently, concomitant with retreatment with Rifaximin 550 mg t.i.d., octreotide 100 mcg subcutaneously once daily at bedtime was initiated to stimulate intestinal motility. Substantial improvements in nausea, abdominal pain and bloating were noted in 2 weeks. 
The rate of enteral feeding was increased to $90-100 \mathrm{ml} /$ hour and she began to tolerate small volumes of oral feeding. A repeat HBT showed no elevation of breath hydrogen above $15 \mathrm{ppm}$ in response to the glucose challenge. A glute-free diet low in Fermentable oligo-, di-, and monosaccharides and polyols (FODMAPs) was implemented to reduce rapid fermentation of carbohydrates by small intestinal bacteria. Octreotide was continued and rifaximin therapy was extended on a 2-week on, 2-week periodic basis to prevent the recurrence of SIBO and the development of bacterial resistance. Unfortunately, her GI symptoms of nausea, abdominal pain and bloating returned 6 weeks later. A repeat glucose HBT showed a hydrogen peak of $110 \mathrm{ppm}$ at 45 minutes after glucose administration suggesting recurrence of SIBO, most likely die to the development of resistance to Rifaximin, Antibiotic therapy was changed from Rifaximin to a combination of Metronidazole $500 \mathrm{mg}$ b.i.d. and Neomycin 500 mg b.i.d. Glucose BHT at 1 week after the switch of antibiotics was negative, indicating successful eradication of SIBO. Her GI symptoms once again improved, and the rate of enteral feeding was increased to $90 \mathrm{cc} /$ hour. Patient was discharged home with octreotide and combined antibiotics of Neomycin and Metronidazole for gut dysmotility and prophylaxis of SIBO. During a follow up outpatient visit 6 weeks after discharge, her GI symptoms remained mild and stable. Enteral feeding was increased to $110 \mathrm{ml}$. hour providing 1700 calories per day, Glucose HBT continued to be normal, indicating SIBO was well controlled with Metronidazole/ Neomycin combination.

\section{Discussion}

Failure of antibiotic treatment and recurrence after successful antibiotic decontamination is common in SIBO, especially when there are predisposing factors [1,2]. A Meta-analysis involving 10 placebo-controlled trials showed pooled breath test normalization rate in SIBO was 51.1\% (95\% confidence interval [CI] 46.7-55.5) with antibiotics, compared with 9.8\% (95\% CI 4.6-17.8) for placebo [3]. In an observational study of rifaximin for the treatment of SIBO, $12.6 \%(10 / 80), 27.5 \%(22 / 80)$, and $43.7 \%$ (35/80) patients showed positive glucose HBT at 3,6 , and 9 months after successful antibiotic treatment [4]. In these patients, identification and treatment of the underlying modifiable causes is critical for successful induction and maintenance of remission of SIBO [2]. Prior to hospitalization, our patient experience severe bloating, nausea, flatulence, abdominal pain and diarrhea, symptoms commonly observed in SIBO. The diagnosis was confirmed with a positive glucose HBT. A two-week course of Rifaximin did not improve her symptoms nor normalized her glucose HBT. Patients with scleroderma may have small bowel dysmotility leading to gut stagnation and bacterial overgrowth $[5,6]$. Manometry showed intermittent low-amplitude burst of uncoordinated contractive activities and infrequent occurrence of phase 3 migrating motor complexes. This may lead to impaired transit and SIBO which is resistant to eradication by antibiotics [7]. In our patient, we showed that acute administration of octreotide 75 mg subcutaneously evoked duodenal phase III complexes that were qualitatively similar to the complexes occurring spontaneously in normal subjects [7].

This was followed by 3 additional duodenal; phase III complexes 3 hours following the administration of octreotide. The enhanced motility may have improved transit and decreased stagnation in our patient. We treated our patient with daily octreotide at bedtime for 3 weeks concurrent with Rifaximin 550 mg three times daily, Substantial improvement of nausea, bloating and abdominal pain were noted. A repeat HBT showed no elevation of breath hydrogen following glucose challenge. This illustrates the importance of restoring intestinal motility in maintaining the effectiveness of Rifaximin in scleroderma patients with intestinal pseudo-obstruction. Abnormal small intestinal motility is one of the most common conditions associated with recalcitrant bacterial overgrowth [2,7]. Therapy with antibiotics for bacterial overgrowth in these patients is usually unsatisfactory. The role of diet restriction such as low FODMAP diet is unclear. Prokinetic agents such as metoclopramide evoke only low-amplitude motor activity in the proximal GI tract. It is less effective in the distal intestine. Cisapride enhances gastric emptying in scleroderma and improves intestinal transit acutely in idiopathic pseudo-obstruction $[8,9]$. There are no reports, however, that Cisapride has prolonged efficacy against pseudo-obstruction in scleroderma. We previously reported octreotide stimulates intestinal motility in normal subjects and in patients with gut dysmotility secondary to connective tissue disorders such as scleroderma [7]. In such patients, the short-term administration of octreotide reduces bacterial overgrowth and improves abdominal symptoms [7]. In our patient, octreotide was effective when combined with rifaximin for the eradication of SIBO.

Rifaximin is among the most effective antibiotics for the treatment of SIBO $[3,10]$. It is a semi-synthetic, nonsystemic, broad-spectrum antibiotic derived from rifamycin with in-vitro activity against Gram-positive, Gram-negative, and anaerobic bacteria [11,12]. Approved by the Food and Drug Administration for traveler's diarrhea, hepatic encephalopathy, and irritable bowel syndrome (IBS) with diarrhea, rifaximin also has been demonstrated to be effective and safe antibiotic treatment for SIBO [10]. A recent meta-analysis of 32 studies involving 1331 patients with SIBO (24 cohort studies and 8 randomized controlled trials) revealed an overall eradication rate of 70.8\% (95\% CI: 61.4-78.2), and $4.6 \%$ adverse events. Symptom improvement rate after successful eradication of SIBO was 67.7\% (95\% CI: 44.7-86.9) [10]. Although its exact mechanisms in the treatment of SIBO remains incompletely defined, rifaximin is minimally absorbed after oral administration, preserving its high concentrations in the intestine; it has a broad spectrum of antibacterial action, yet minimally alters the gut microbiota and increase the relative abundance of beneficial bacteria, such as Bifidobacteria and Lactobacilli [12-15]. These actions may reduce bacterial fermentation of carbohydrates, 
improve intestinal permeability and decrease low grade mucosal inflammation. contributing to the beneficial effect of rifaximin in SIBO $[13,14]$.

Unfortunately, despite initial success of treating her SIBO, the patient's abdominal pain and bloating subsequently worsened after 6 weeks use of rifaximin, accompanied by positive glucose hydrogen breath test, suggesting the development of rifaximin resistance in her intestinal flora. We discontinued Rifaximin and started her with a combination of Metronidazole and Neomycin. Within 1 week her glucose hydrogen breath test returned to normal, accompanied by marked improvement in bloating, abdominal pain and nausea. Over the last few months, our patient has continued to do well with rotating antibiotic regimens and daily octreotide to stimulate intestinal motility. Resistance to rifaximin is relatively rare because this condition develops from a stable mutation in the host cell DNA, thus unlike plasmid-mediated antibiotic resistance, rifaximin resistance does not transmit from one strain to another [16]. In cirrhotic patients with hepatic encephalopathy (HE), long-term rifaximin treatment for HE was not associated with development of rifaximin resistance or increase in the rate of infections [17]. Recent studies report that E. Coli develops rifaximin resistance in populations subjected to an environmental selective pressure [18]. Another study shows that skin rifampin-resistant staphylocose emerge after prolonged intake of Rifaximin [19]. To date there is no report on the development of Rifaximin resistance in the treatment of SIBO.

\section{Conclusion}

We reported a case of scleroderma with small bowel dysmotility and bacterial overgrowth. On different occasions, our patient ailed to respond to Rifaximin, one of the most commonly used antibiotics for SIBO. Our case demonstrated that restoring small bowel motility is critical to maintain the effectiveness of Rifaximin in the treatment of SIBO. The case also shows that prolonged use of Rifaximin may result in the development of Rifaximin resistant bacteria, a condition rarely reported. Rotating antibiotic regimens is important for treating challenging case of SIBO requiring prolonged use of antibiotics.

\section{References}

1. Kahn IJ, Jeffries GH, Sleisenger MH (1966) Malabsorption in intestinal scleroderma. Correction by antibiotics. N Engl J Med 274(24): 13391344.

2. Rezaie A, Pimentel M, Rao SS (2016) How to test and treat small intestinal bacterial overgrowth: An evidence-based approach. Curr Gastroenterol Rep 18(2): 8.
3. Shah SC, Day LW, Somsouk M, Sewell JL (2013) Meta-analysis: Antibiotic therapy for small intestinal bacterial overgrowth. Aliment Pharmacol Ther 38(8): 925-934.

4. Lauritano EC, Gabrielli M, Scarpellini E, Lupascu A, Novi M, et al. (2008) Small intestinal bacterial overgrowth recurrence after antibiotic therapy. Am J Gastroenterol 103(8): 2031-2035.

5. Peachey RD, Creamer B, Pierce JW (1969) Sclerodermatous involvement of the stomach and the small and large bowel. Gut 10(4): 285-292.

6. Aufdermaur M (1981) Juvenile kyphosis (Scheuermann's disease): Radiography, histology, and pathogenesis. Clin Orthop Relat Res 154: 166-174.

7. Soudah HC, Hasler WL, Owyang C (1991) Effect of octreotide on intestinal motility and bacterial overgrowth in scleroderma. $\mathrm{N}$ Engl J Med 325(21): 1461-1467.

8. Horowitz M, Maddern GJ, Maddox A, Wishart J, Chatterton BE, et al. (1987) Effects of cisapride on gastric and esophageal emptying in progressive systemic sclerosis. Gastroenterology 93(2): 311-315.

9. Camilleri M, Malagelada JR, Abell TL, Brown ML, Hench V, et al. (1989) Effect of six weeks of treatment with cisapride in gastroparesis and intestinal pseudoobstruction. Gastroenterology 96(3): 704-712.

10. Gatta L, Scarpignato C (2017) Systematic review with meta-analysis: Rifaximin is effective and safe for the treatment of small intestine bacterial overgrowth. Aliment Pharmacol Ther 45(5): 604-616.

11. Menees SB, Maneerattannaporn M, Kim HM, Chey WD (2012) The efficacy and safety of rifaximin for the irritable bowel syndrome: a systematic review and meta-analysis. Am J Gastroenterol 107(1): 28-35.

12. Adachi JA, DuPont HL (2006) Rifaximin: a novel nonabsorbed rifamycin for gastrointestinal disorders. Clin Infect Dis 42(4): 541-547.

13. Ponziani FR, Scaldaferri F, Petito V, Paroni Sterbini F, Pecere S, et al. (2016) The Role of Antibiotics in Gut Microbiota Modulation: The Eubiotic Effects of Rifaximin. Dig Dis 34(3): 269-278.

14.Xu D, Gao J, Gillilland M, Wu X, Song I, et al. (2014) Rifaximin alters intestinal bacteria and prevents stress-induced gut inflammation and visceral hyperalgesia in rats. Gastroenterology 146(2): 484-496 e484.

15. Jiang ZD, DuPont HL (2005) Rifaximin: in vitro and in vivo antibacterial activity--A review. Chemotherapy 51 Suppl 1: 67-72.

16. Food Drug Administration (2013) Xifaxan (rifaximin) tablets, $550 \mathrm{mg}$ NDA 21-361: Briefing document for gastrointestinal drugs advisory committee meeting 16 November 2011.

17. Mullen KD, Sanyal AJ, Bass NM, Poordad FF, Sheikh MY, et al. (2014) Rifaximin is safe and well tolerated for long-term maintenance of remission from overt hepatic encephalopathy. Clin Gastroenterol Hepatol 12(8): 1390-1397.

18. Gomes C, Ruiz L, Pons MJ, Ochoa TJ, Ruiz J (2013) Relevant role of efflux pumps in high levels of rifaximin resistance in Escherichia coli clinical isolates. Trans R Soc Trop Med Hyg 107(9): 545-549.

19. Valentin T, Leitner E, Rohn A, Zollner-Schwetz I, Hoenigl M, et al. (2011) Rifaximin intake leads to emergence of rifampin-resistant staphylococci. J Infect 62(1): 34-38. 
(c) (P) This work is licensed under Creative

To Submit Your Article Click Here: Submit Article

DOI: 10.32474/CTGH.2018.01.000115

$\begin{gathered}\text { Current Trends in Gastroenterology } \\ \text { and Hepatology }\end{gathered}$
Assets of Publishing with us
- Global archiving of articles
- Immediate, unrestricted online access
- Rigorous Peer Review Process
- Authors Retain Copyrights
- Unique DOI for all articles

